\title{
Histopathological expression analysis of intercellular adhesion molecule 1 (ICAM-1) along development and progression of human melanoma
}

\author{
Gilli Galore-Haskel ${ }^{1, *}$, Erez N. Baruch ${ }^{1,2, *}$, Amy L. Berg ${ }^{1}$, Iris Barshack ${ }^{3,4}$, Itzhak \\ Zilinsky ${ }^{4,5}$, Camila Avivi ${ }^{3}$, Michal J. Besser ${ }^{1,2}$, Jacob Schachter ${ }^{1,4}$ and Gal Markel ${ }^{1,2,6}$ \\ ${ }^{1}$ Ella Lemelbaum Institute of Immuno-Oncology, Ramat-Gan, Israel \\ ${ }^{2}$ Clinical Microbiology and Immunology, Tel-Aviv, Israel \\ ${ }^{3}$ Institute of Pathology, Ramat-Gan, Israel \\ ${ }^{4}$ School of Medicine, Sackler Faculty of Medicine, Tel Aviv University, Tel-Aviv, Israel \\ ${ }^{5}$ Department of Plastic and Reconstructive Surgery, Ramat-Gan, Israel \\ ${ }^{6}$ Talpiot Medical Leadership Program, Sheba Medical Center, Ramat-Gan, Israel \\ *These authors have contributed equally to this work
}

Correspondence to: Gal Markel, email: Markel@post.tau.ac.il

Keywords: melanoma, ICAM-1, adhesion, progression

Received: April 12, $2017 \quad$ Accepted: July 11, $2017 \quad$ Published: September 14, 2017

Copyright: Galore-Haskel et al. This is an open-access article distributed under the terms of the Creative Commons Attribution License 3.0 (CC BY 3.0), which permits unrestricted use, distribution, and reproduction in any medium, provided the original author and source are credited.

\section{ABSTRACT}

Intercellular adhesion molecule 1 (ICAM-1) protein is an important adhesion molecule that facilitates metastasis on one hand, and on the other hand supports the immunological synapse necessary for T-cell mediated elimination. The expression pattern of ICAM-1 in melanoma was studied more than two decades ago, mainly in cell lines or in unmatched melanoma specimens. By using real time PCR we could not demonstrate a clear difference in ICAM-1 mRNA levels between primary melanocytes and primary cultures of metastatic melanoma. However, immunohistochemistry staining of progression tissue microarray comprised of samples of different disease stages derived from different patients, demonstrated a dramatic ICAM-1 upregulation particularly upon the transition from primary tumor to lymph node metastasis. There was no significant difference between lymph node and distant metastases. Importantly, these results were confirmed in an independent tissue microarray comprised of patient-paired specimens from progressive stages of the patient's disease. These data indicate that ICAM-1 upregulation is required to initiate the lymphatic spread of melanoma (Stage III) but no further increase is associated with progression to remote organs (Stage IV).

\section{INTRODUCTION}

Malignant melanoma, arising from pigment producing melanocytes, is the most lethal form of skin cancer. The incidence of melanoma in Caucasian populations has been increasing at a higher rate than any other malignancy [1]. Numerous molecular events have been associated with the development and progression of melanoma by affecting different pathways for proliferation, apoptosis and migration. (reviewed in [2]).
Cell adhesion molecules (CAMs) are cell surface molecules which assist cells to adhere to other cells or to the extracellular matrix. They also allow the exchange of information between cells. Alterations in the function and expression of CAMs results in disruption of normal cell-cell interactions and may lead to malignant transformation and tumor progression (reviewed in [3]). Human melanoma cells have been found to express a number of cell adhesion molecules, among them Intercellular adhesion molecule 1 (ICAM-1), which mediates their interaction with leukocytes [4]. 
Binding of ICAM-1 to integrin lymphocyte functionassociated antigen-1 (LFA-1) is essential for optimal interaction between CTLs and target cells and facilitates T-cell activation [5]. Pandolfi et al. showed that antiICAM-1 inhibited the cytotoxicity of tumor infiltrating lymphocytes against autologous melanoma cells [6]. ICAM-1 can be induced by inflammatory cytokines such as IFN- $\gamma$, IL- 1 and TNF- $\alpha$ [7]. We have previously shown that melanoma cells downregulate the expression of adenosine deaminase acting on RNA (ADAR1) enzyme upon the transition from primary to metastatic melanoma [8]. This renders the melanoma cells more resistant to T-cells by lowering ICAM-1 protein expression [9]. On the other hand, several studies suggested the involvement of ICAM-1 in melanoma progression and prognosis. The expression of ICAM-1 by cells of melanocytic origin increases with the progression of the malignant transformation process [10-13], and a significant association was observed between ICAM-1 expression in primary lesions and the thickness of the lesion as well as with a reduction in disease free survival $[11,14]$. In line with these studies, in vivo studies showed that suppression of ICAM-1 expression inhibits the metastatic capacity of melanoma cells [15]. Importantly, the expression of ICAM-1 in melanocytic lesions was last studied in 1997, using primarily cell lines which may be significantly biased, and may account for the conflicting data described above. In this work, we analyze the expression of ICAM-1 during melanoma development and progression using low passage primary melanocyte and melanoma cultures and two progression tissue microarrays.

\section{RESULTS}

To recapitulate previous similar studies, with the exception of focusing mostly on low passage cultures, the expression of ICAM-1 in metastatic melanoma cells was assessed by qRT-PCR using 15 low-passage patient-derived metastatic melanoma cultures, 3 metastatic melanoma cells lines and 3 cultures of normal melanocytes. Similarly to the equivocal available data from the literature, ICAM-1 expression was heterogeneously expressed in all three categories without any clear trend (Figure 1). Notably, mRNA expression analysis cannot distinguish between cytoplasmic and membrane expression.

ICAM-1 is an intercellular molecule, which exerts its role by binding to its receptor LFA-1. To take into account cellular location and avoid culture biases, we profiled membrane expression by using the progression TMA which was purchased from the NCI. Due to technical reasons (i.e., folded specimens or tissue loss), only 75 benign nevi, 61 primary tumors, 37 lymph node metastases and 55 metastases from other locations were analyzed for ICAM-1 immuno-staining intensity. ICAM-1 expression profile in this TMA is illustrated in Figure 2A. Clearly, staining intensity increases with disease progression. For example, specimens with high intensity rates comprised only $7 \%$ of the nevi, as compared to $51 \%$ of the distant metastases. The increase in ICAM-1 expression is statistically significant. While ICAM-1 expression in primary tumors was similar to that of nevi $(p=0.44)$, it was lower than ICAM-1 expression in both lymph node and in distant metastases $(p<0.001$ for either). ICAM-1 expression in distant metastases demonstrated a trend towards a higher level than in lymph node metastases which was not significant statistically $(p=0.09)$. These data point to the progressive upregulation of ICAM-1 expression, which is particularly evident upon the initial regional spread to the lymph nodes. Representative staining patterns of specimens $(\times 4)$ are shown in Figure 2B.

We further tested ICAM-1 expression on our inhouse designed TMA of paired melanoma specimens from different disease stages, in order to neutralize potential bias of inter-patient variations. Due to uninterpretable spots, staining results of the entire progression spectrum (primary tumor, lymph node metastasis and distant metastasis) were available for only 7 patients of the original 12 . Patients with partial spectrum included 3 patients who used to have full progression spectrum and 2 patients who originally had only partial spectrum. Two patients who originally had full spectrum were excluded due to uninterpretable spots for 2 or more samples. Overall, the histopathology slide represented 12 patients with 33 samples out of the original $42(79 \%)$. A $21 \%$ sample loss rate is similar to those reported by others ( $10 \%$ to $\sim 20 \%$ ) [16]. The clinical and pathological characteristics for these 12 patients are summarized in Table 1.

As illustrated in Figure 3A, lymph node metastases had a significantly higher ICAM-1 expression than primary tumors $(p=0.02)$. Interestingly, lymph node metastases seem to have higher ICAM-1 expression than distant metastases but this difference was not statistically significant $(p=0.068)$. These results concur with those observed with the TMA purchased from the NCI. A representative staining of all three disease stages from one patient is shown in Figure 3B. There was no significant difference in ICAM-1 intensity between primary tumors of cutaneous or mucosal origin $(p=0.8)$. Additionally, there was no significant difference in ICAM-1 intensity in distant metastases in soft tissue versus visceral organs $(p=0.4)$. Finally, an inverse correlation was suggested between ICAM-1 intensity on primary or distant metastasis and progression to Stage III or to death, respectively ( $p=0.075$ and $p=0.08$, respectively). ICAM-1 intensity on lymph node metastases was not significantly associated with progression to Stage IV or survival ( $p=0.13$ and $p=0.34$, respectively). 
Table 1: Clinical-pathological characteristics of patients comprising the patient-paired TMA

\begin{tabular}{|c|c|c|c|c|c|c|c|c|c|c|}
\hline $\begin{array}{l}\text { Patient } \\
\text { number }\end{array}$ & Age & Gender & $\begin{array}{l}\text { BRAF } \\
\text { mutation } \\
\text { status }\end{array}$ & $\begin{array}{l}\text { Ulceration } \\
\text { of primary } \\
\text { tumor }\end{array}$ & $\begin{array}{c}\text { Location of } \\
\text { primary tumor }\end{array}$ & $\begin{array}{c}\text { Location of lymph } \\
\text { node metastases }\end{array}$ & $\begin{array}{c}\text { Progression from } \\
\text { primary to LN } \\
\text { metastases (months) }\end{array}$ & $\begin{array}{l}\text { Location of distant } \\
\text { metastases }\end{array}$ & $\begin{array}{c}\text { Progression from } \\
\text { LN metastases to } \\
\text { distant metastases } \\
\text { (months) }\end{array}$ & $\begin{array}{r}\text { Overall } \\
\text { survival } \\
\text { (months) }\end{array}$ \\
\hline 1 & 66 & $\mathrm{~F}$ & WT & $\mathrm{Y}$ & Vulva & Inguinal & 0 & Small bowel & -9.1 & 35.8 \\
\hline 2 & 70 & $\mathrm{~F}$ & WT & $\mathrm{N}$ & Upper back & Axilla & 0 & Sub-cutaneous & 16.5 & 34 \\
\hline 3 & 83 & $\mathrm{~F}$ & WT & UNK & Leg & Thigh & 16 & Sub-cutaneous & 1.2 & 37.2 \\
\hline 4 & 34 & M & WT & $\mathrm{Y}$ & Leg & Inguinal & 1 & Sub-cutaneous & 40.7 & 99.5 \\
\hline 5 & 59 & M & WT & UNK & Conjunctiva & Neck & 17.7 & Retroperitoneum & 14.5 & 51.1 \\
\hline 6 & 60 & M & UNK & UNK & Flank & Axilla & 13.4 & $\begin{array}{c}\text { Supraclavicular } \\
\text { LN }\end{array}$ & 0 & 23.7 \\
\hline 7 & 71 & $M$ & WT & UNK & Shoulder & Axilla & 0 & Muscle & 3.9 & 11.7 \\
\hline 8 & 62 & $\mathrm{~F}$ & UNK & $\mathrm{Y}$ & Flank & Axilla & 12.2 & Sub-cutaneous & -12.17 & 17.2 \\
\hline 9 & 51 & $\mathrm{~F}$ & UNK & Y & Buttocks & Inguinal & 0.63 & Sub-cutaneous & 3.3 & 16 \\
\hline 10 & 43 & $\mathrm{~F}$ & WT & Y & Vagina & Inguinal & 5.7 & Sub-cutaneous & 0 & 24.2 \\
\hline 11 & 85 & $\mathrm{M}$ & WT & $\mathrm{Y}$ & Scalp & Neck & 1.9 & Salivary gland & 10.1 & 47.2 \\
\hline 12 & 50 & $\mathrm{~F}$ & WT & Y & Vulva & Inguinal & 99.4 & Lung & -59.9 & $\begin{array}{l}\text { Alive } \\
\text { for } 151 \\
\text { months }\end{array}$ \\
\hline
\end{tabular}

WT stands for Wild Type; UNK stands for Unknown; LN stands for Lymph Nodes.

F-female, M-male.

\section{DISCUSSION}

ICAM-1 expression has been shown to correlate with the metastatic potential of melanoma. Our results show the expression of ICAM-1 at the mRNA level in cultured primary melanoma and cell lines, as well as in melanocytes, is heterogeneous with no significant difference in ICAM-1 expression between benign and

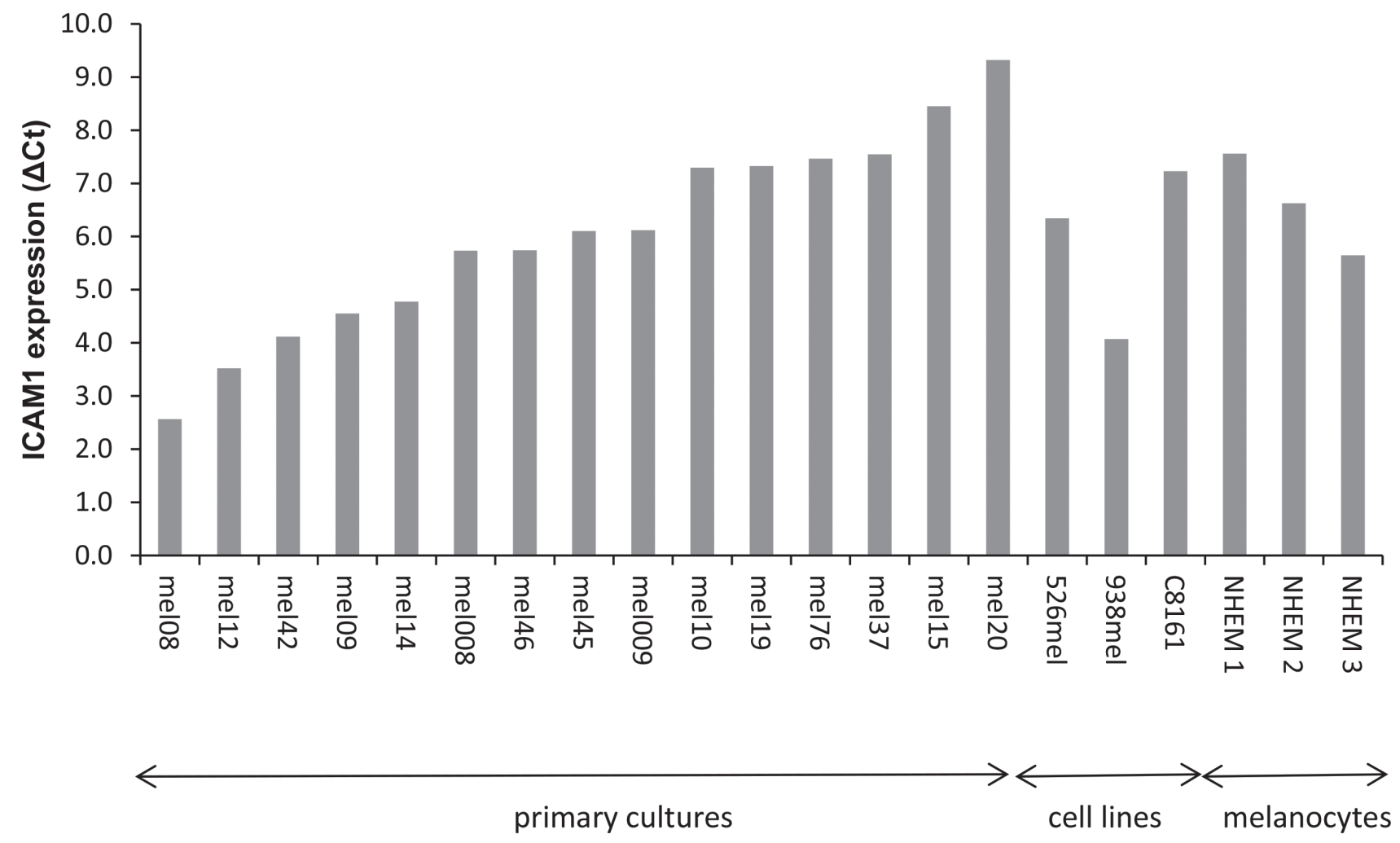

Figure 1: ICAM-1 expression in cultured melanocytes and melanoma cells. The expression of ICAM-1 was tested with realtime quantitative PCR in 15 low-passage patient-derived metastatic melanoma cultures, 3 melanoma cell lines and 3 cultures of normal melanocytes. Results are expressed as $\Delta \mathrm{Ct}$, normalized to GAPDH expression. 
malignant lesions (Figure 1). However, at the protein level, a significant increase in ICAM-1 membrane expression along the metastatic progression is observed in two independent TMAs (Figures 2-3). This discrepancy could be explained by the different specimens analyzed (i.e., cultured cells versus paraffin embedded tissues) as

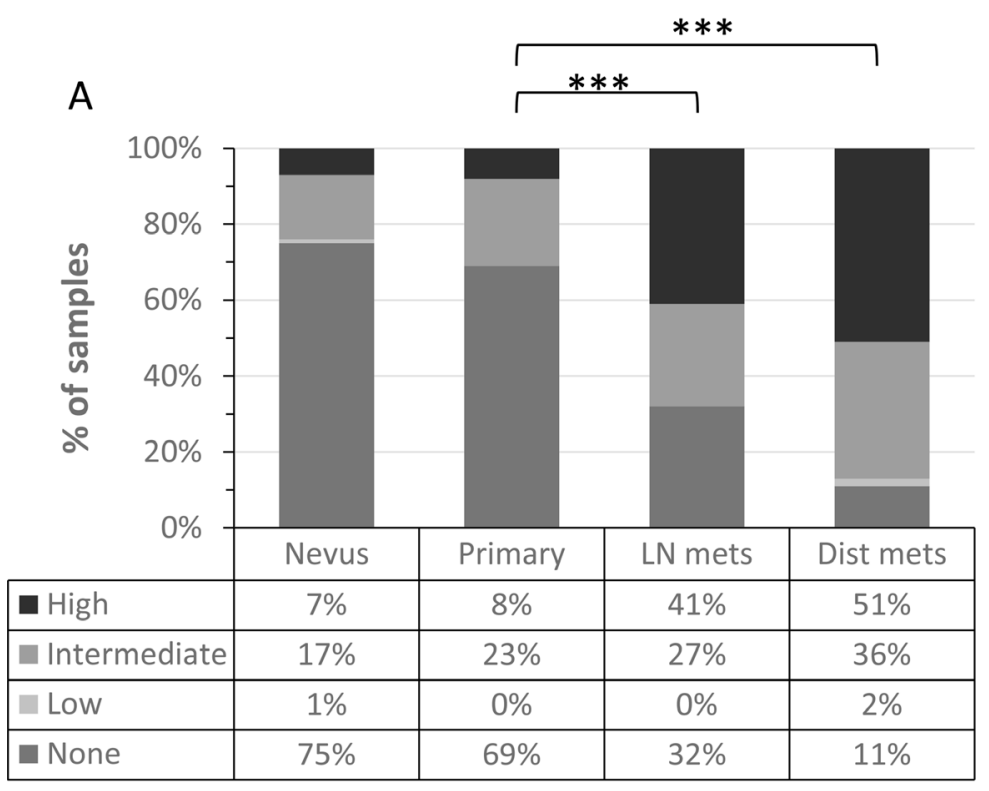

B

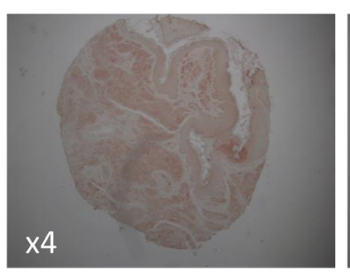

Nevus (low)

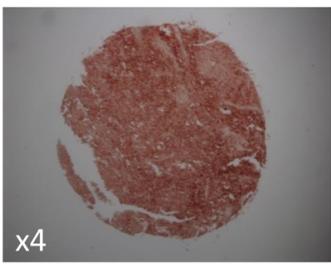

Lymph node metastasis (high)

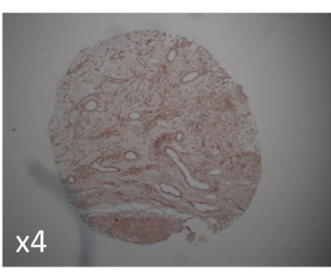

Primary (intermediate)

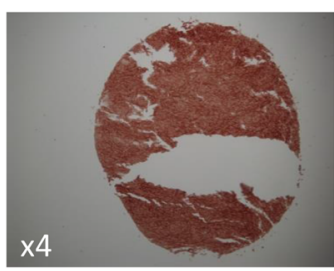

Distant metastasis (high)

Figure 2: ICAM-1 expression with melanoma development and progression. (A) ICAM-1 membrane expression was analyzed in melanoma progression TMA comprised of nevi, primary tumors, lymph node (LN) metastases and distant metastases. Intensity staining of ICAM-1 was scored as none, low, intermediate or high; (B) Representative staining patterns $(\times 4)$ of ICAM-1 in melanocytic specimens.

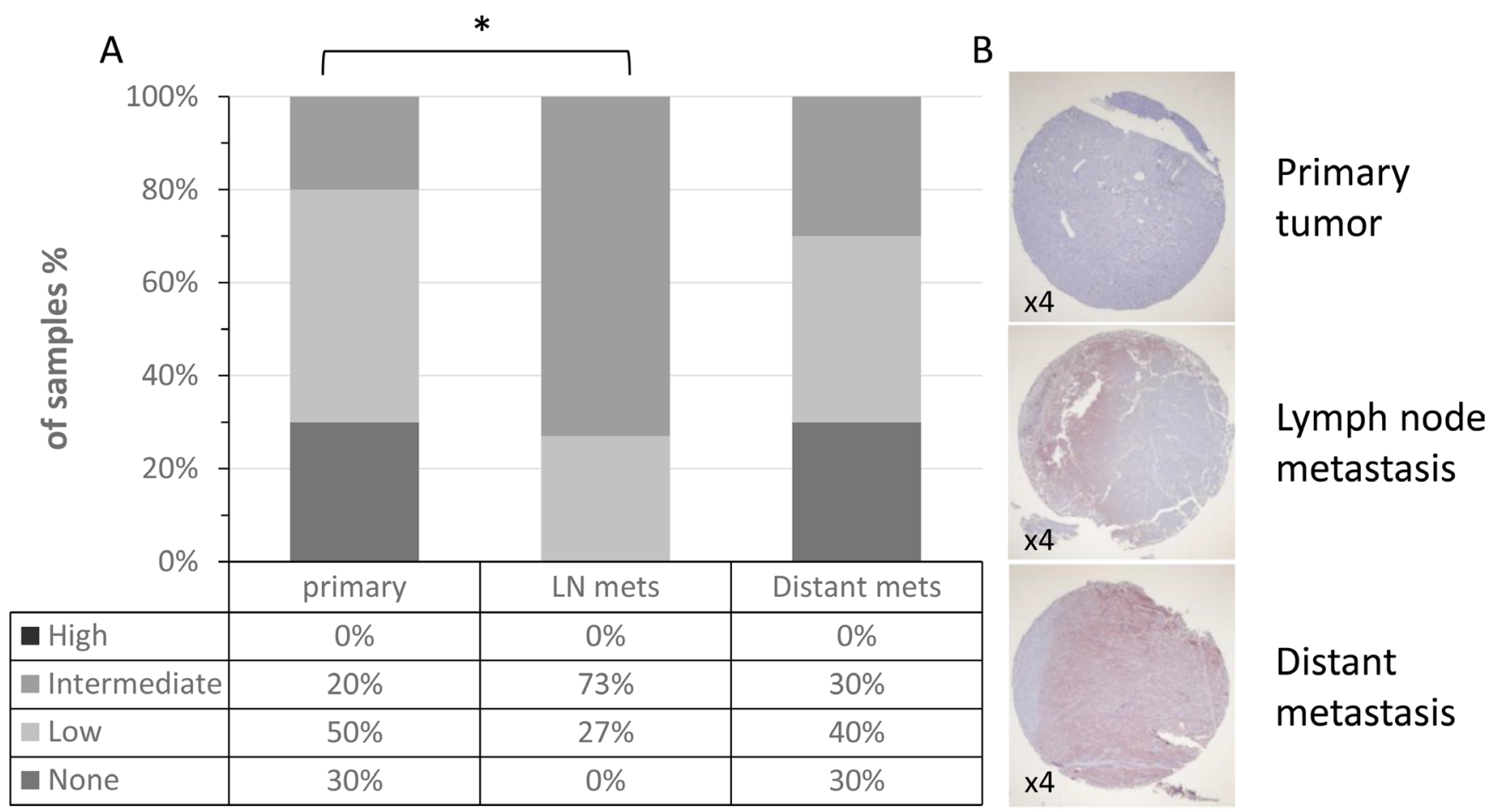

Figure 3: ICAM-1 expression in patient paired samples of disease progression. (A) ICAM-1 membrane expression was analyzed in our in-house TMA comprised of paired primary tumors, lymph node (LN) metastases and distant metastases. Intensity staining of ICAM-1 was scored as none, low, intermediate or high. (B) Representative staining patterns $(\times 4)$ of ICAM-1 in melanocytic specimens. 
well as by the different detection methods applied (i.e., qRT-PCR versus immunohistochemistry). Protein but not mRNA level studies take into account post-transcriptional and post-translational processes, thus the results of the immunohistochemistry are more reliable. Even though the mRNA and IHC studies were not done on the same samples, the substantial amount of cases in the TMA from the NCI and the pairing of samples in our in-house TMA provide statistical confidence for the validity of this observation.

Our findings are in accordance with previous reports showing higher ICAM-1 expression in metastases than in benign and primary lesions $[11,14]$. The level of ICAM-1 expression on melanoma cells correlates with tumor thickness and risk of metastases [10-14]. Here we specifically show that ICAM-1 is mostly upregulated in the first site of metastasis, i.e. the lymph node, with no further statistically significant increase in ICAM-1 expression in distant metastases. This suggests that melanoma cells upregulate ICAM-1 in early stages of metastasis to enable at least regional spread. This progressive upregulation is confirmed for the first time in individual paired samples. Indeed, we observed a potential link between the expression intensity of ICAM-1 in primary melanoma and progression to Stage III. There was no association between ICAM-1 in lymph node metastases and progression to Stage IV or overall survival. An additional potential link between ICAM-1 in distant metastasis and lower survival was suggested. It is still unclear why there may be an association between prognosis and ICAM-1 expression on distant metastases but not lymph node metastases. The main study limitation is the small number of included patients. This could highlight the effect of certain subpopulations, for example: a) 9 of 12 samples are BRAF wild type, with the BRAF status of the remaining three unknown; or b) ulcerated primary, which is represented here in 7 of 12 specimens. It should be mentioned that our confirmatory studies were done with different antibodies as compared to earlier studies. In addition, the studies performed on the TMA obtained from the NCI and the patient-matched TMA we generated, were analyzed using anti ICAM-1 antibodies of different lots. This may account for the different intensities reported for the TMAs.

These results may seem to contradict the fact that ICAM-1 strengthens CTL-melanoma cell interactions which would lead to more efficient elimination of the tumor cells and better prognosis [17, 18]. Several possible mechanisms have been suggested to explain this contradiction; ICAM-1 on melanoma cells forms aggregates with leukocytes, allowing their dissociation from the tumor and ultimately their metastatic spread $[19,20]$. An alternative explanation is that soluble ICAM1 , shed from melanoma cells, competes with membranebound ICAM-1, thus preventing the interaction and destruction of tumor cells by effector cells [21, 22].

\section{MATERIALS AND METHODS}

\section{Cells}

The melanoma lines $526 \mathrm{mel}$ and $938 \mathrm{mel}$ (obtained from Dr. Steven Rosenberg, National Cancer Institute, Bethesda, MD), C8161 (obtained from Dr. Marry Hendrix, Children's Memorial Research Center, Chicago, IL) and normal human epidermal melanocytes (NHEM; PromoCell, Heidelberg, Germany) were maintained as previously described [8]. The 15 primary cultures derived from surgically removed metastatic melanoma specimens were established and cultured as previously described [23].

\section{RNA isolation and reverse transcription}

Total RNA was isolated from melanoma lines, melanocytes and primary cultures using Tri Reagent (Sigma-Aldrich, Rehovot, Israel), and cDNA was generated by Universal Transcriptor cDNA master (Roche Diagnostics, Basel, Switzerland), according to the manufacturer's instructions.

\section{Quantitative real-time PCR (qRT-PCR)}

Primers (Sigma-Aldrich) were designed according to Primer-Express software guidelines (Applied Biosystems). The qRT-PCR reactions were run in triplicates LightCycler 480 system (Roche). Gene transcripts were detected using LightCycler 480 SYBR Green I Master (Roche), according to the manufacturer's instructions. Reactions were normalized to GAPDH endogenous control. Expression was calculated as $\Delta \mathrm{Ct}$. The detailed sequences of primers used: ICAM-1-F 5'-TGCAGACAGTGACCATCTACAGC; ICAM-1-R 5'-TCACCTCGGTCCCTTCTGAG; GAPDH-F 5'-TGCACCACCAACTGCTTAGC; GAPDH-R 5'-GGCAT GGACTGTGGTCATGAG.

\section{Progression TMA}

Progression tissue microarray (TMA) slides were provided by the NCI CDP and included 98 benign nevi, 73 primary tumors, and 41 lymph node metastases and 72 metastases from other locations. Other investigators may have received slides from these same array blocks. Immunohistochemical staining was performed on the TMA samples using a commercially available polyclonal rabbit anti-ICAM-1 antibody (Prestige Antibodies, Sigma Aldrich, Rehovot, Israel) according to standard procedures. A blinded assessment of ICAM-1 expression was conducted by an expert pathologist (IB). For each sample, intensity of ICAM-1 membrane expression was scored as none, low intensity, intermediate or high. Digital images were captured with Olympus BX51 microscope. 


\section{Progression TMA of paired samples}

Progression TMA of paired samples from the same patient was designed in-house as a template for the assessment of the melanoma progression process. Formalin-fixed, paraffin-embedded paired tissue samples of primary tumors, lymph node metastases and distant metastases were collected from 12 patients. Paired samples from a patient with a primary tumor and a lymph metastasis, and another patient with lymph node and distant metastases, were further included along with seven normal liver tissue samples and 3 normal muscle tissue samples which were used for orientation and control. Each tissue sample was initially stained with Hematoxylin and Eosin (H\&E) and representative areas of tumors were marked by an expert pathologist (IB). Accordingly, three $2 \mathrm{~mm}$ diameter tissue cylinders were punched out from each tumor block. The cylinders were deposited into a recipient block using Manual Tissue Arrayer MTA-1 (Beecher Instruments Inc., Sun Prairie, WI, USA). Tumor sample triplicates were used as a means of overcoming tumor heterogeneity, as triplicate arrayed-derived data demonstrate a concordance of over 95\% with full-section-derived data [24]. Post array construction, a $4 \mu \mathrm{m}$ section was $\mathrm{H} \& \mathrm{E}$ stained to confirm the histological quality. A consecutive $4 \mu \mathrm{m}$ section was used for immunohistochemical staining using anti-ICAM-1 antibody, as described above. Each spot was scored by a blinded expert pathologist (IB) according to the same staining intensity scale used in the progression TMA, i.e. none, low intensity, intermediate or high. Uninterpretable cores due to loss of the tissue or excessive background staining were excluded from the analyses.

\section{Ethics}

All studies involving patient-derived material were approved by the Institutional Review Board of Sheba Medical Center.

\section{Statistical analysis}

The Mann Whitney Test was used to analyze the data of TMA purchased from the NCI, while the Wilcoxon Signed Rank Test, was used to analyze the data of the patient-paired TMA. Additionally, for the paired-TMA, the Mann Whitney Test was used to assess the correlation between staining intensity and tumor site while the oneway ANOVA test was used to assess the correlation between staining intensity and time to disease progression. Statistical tests were performed with STATA (STAT Corp, STATA Statistics/Data analysis for Windows, Version 15.0, College Station, Texas, USA). Significance was defined as a $P$ value of $<0.05$.

\section{ACKNOWLEDGMENTS AND FUNDING}

GM is supported by grants from the Israel Science Foundation, the Israel Ministry of Economy and the Israel Cancer Association. The authors would like to thank Chaya and Nehemia Lemelbaum for their generous support.

\section{CONFLICTS OF INTEREST}

None.

\section{REFERENCES}

1. Berwick M, Erdei E, Hay J. Melanoma epidemiology and public health. Dermatol Clin. 2009; 27:205-14, viii. https:// doi.org/10.1016/j.det.2008.12.002S0733-8635(08)00111-3.

2. Shain AH, Bastian BC. From melanocytes to melanomas. Nat Rev Cancer. 2016. https://doi.org/10.1038/ nrc.2016.37nrc.2016.37.

3. Wai Wong C, Dye DE, Coombe DR. The role of immunoglobulin superfamily cell adhesion molecules in cancer metastasis. Int J Cell Biol. 2012; 2012:340296. https://doi.org/10.1155/2012/340296.

4. Johnson JP. Cell adhesion molecules of the immunoglobulin supergene family and their role in malignant transformation and progression to metastatic disease. Cancer Metastasis Rev. 1991; 10:11-22.

5. Bachmann MF, McKall-Faienza K, Schmits R, Bouchard D, Beach J, Speiser DE, Mak TW, Ohashi PS. Distinct roles for LFA-1 and CD28 during activation of naive T cells: adhesion versus costimulation. Immunity. 1997; 7:549-57. https://doi.org/S1074-7613(00)80376-3.

6. Pandolfi F, Trentin L, Boyle LA, Stamenkovic I, Byers HR, Colvin RB, Kurnick JT. Expression of cell adhesion molecules in human melanoma cell lines and their role in cytotoxicity mediated by tumor-infiltrating lymphocytes. Cancer. 1992; 69:1165-73.

7. Maio M, Gulwani B, Tombesi S, Ferrone S. Modulation by cytokines of HLA antigens, intercellular adhesion molecule 1 and high molecular weight melanoma associated antigen expression and of immune lysis of clones derived from the melanoma cell line MeM 50-10. Cancer Immunol Immunother. 1989; 30:34-42.

8. Nemlich Y, Greenberg E, Ortenberg R, Besser MJ, Barshack I, Jacob-Hirsch J, Jacoby E, Eyal E, Rivkin L, Prieto VG, Chakravarti N, Duncan LM, Kallenberg DM, et al. MicroRNA-mediated loss of ADAR1 in metastatic melanoma promotes tumor growth. The Journal of clinical investigation. 2013; 123:2703-18. https://doi.org/10.1172/JCI62980.

9. Galore-Haskel G, Nemlich Y, Greenberg E, Itzhaki O, Shoshani N, Shapira-Fromer R, Ben-Ami E, Besser MJ, Schachter J, Markel G. A novel immune resistance 
mechanism of melanoma cells controlled by the ADAR1 enzyme. Oncotarget. 2015; 6:28999-9015. https://doi. org/10.18632/oncotarget.4905.

10. Natali PG, Hamby CV, FeldingHabermann B, Liang BT, Nicotra MR, DiFilippo F, Giannarelli D, Temponi M, Ferrone S. Clinical significance of alpha(v)beta(3) integrin and intercellular adhesion molecule-1 expression in cutaneous malignant melanoma lesions. Cancer Research. 1997; 57:1554-60.

11. Natali P, Nicotra MR, Cavaliere R, Bigotti A, Romano G, Temponi M, Ferrone S. Differential expression of intercellular adhesion molecule 1 in primary and metastatic melanoma lesions. Cancer Res. 1990; 50:1271-8.

12. Johnson JP, Stade BG, Holzmann B, Schwable W, Riethmuller G. De novo expression of intercellular-adhesion molecule 1 in melanoma correlates with increased risk of metastasis. Proc Natl Acad Sci USA. 1989; 86:641-4.

13. Si Z, Hersey P. Immunohistological examination of the relationship between metastatic potential and expression of adhesion molecules and 'selectins' on melanoma cells. Pathology. 1994; 26:6-15.

14. Kageshita T, Yoshii A, Kimura T, Kuriya N, Ono T, Tsujisaki M, Imai K, Ferrone S. Clinical relevance of ICAM-1 expression in primary lesions and serum of patients with malignant melanoma. Cancer Res. 1993; 53:4927-32.

15. Miele ME, Bennett CF, Miller BE, Welch DR. Enhanced metastatic ability of TNF-alpha-treated malignant melanoma cells is reduced by intercellular adhesion molecule-1 (ICAM-1, CD54) antisense oligonucleotides. Experimental Cell Research. 1994; 214:231-41. https://doi. org/S0014-4827(84)71253-510.1006/excr.1994.1253.

16. Hager M, Kolbitsch C, Tiefenthaler W, Haufe H, Kemmerling R, Lucia Moser P. Tissue microarrays from renal cell tumors: exclusion criteria and rate of exclusion. Scand J Urol Nephrol. 2007; 41:485-9. https://doi. org/10.1080/00365590701520552.

17. Webb DS, Mostowski HS, Gerrard TL. Cytokine-induced enhancement of ICAM-1 expression results in increased vulnerability of tumor cells to monocyte-mediated lysis. J Immunol. 1991; 146:3682-6.
18. Jenkinson SR, Williams NA, Morgan DJ. The role of intercellular adhesion molecule-1/LFA-1 interactions in the generation of tumor-specific CD8+ T cell responses. J Immunol. 2005; 174:3401-7. https://doi.org/174/6/3401.

19. Liang S, Slattery MJ, Wagner D, Simon SI, Dong C. Hydrodynamic shear rate regulates melanoma-leukocyte aggregation, melanoma adhesion to the endothelium, and subsequent extravasation. Ann Biomed Eng. 2008; 36:661-71. https://doi.org/10.1007/s10439-008-9445-8.

20. Liang S, Hoskins M, Dong C. Tumor cell extravasation mediated by leukocyte adhesion is shear rate dependent on IL-8 signaling. Mol Cell Biomech. 2010; 7:77-91.

21. Altomonte M, Gloghini A, Bertola G, Gasparollo A, Carbone A, Ferrone S, Maio M. Differential expression of cell adhesion molecules CD54/CD11a and CD58/ CD2 by human melanoma cells and functional role in their interaction with cytotoxic cells. Cancer Res. 1993; 53:3343-8.

22. Becker JC, Termeer C, Schmidt RE, Brocker EB. Soluble intercellular adhesion molecule-1 inhibits MHC-restricted specific $\mathrm{T}$ cell/tumor interaction. J Immunol. 1993; 151:7224-32.

23. Besser MJ, Shapira-Frommer R, Treves AJ, Zippel D, Itzhaki O, Hershkovitz L, Levy D, Kubi A, Hovav E, Chermoshniuk N, Shalmon B, Hardan I, Catane R, et al. Clinical responses in a phase II study using adoptive transfer of short-term cultured tumor infiltration lymphocytes in metastatic melanoma patients. Clin Cancer Res. 2010; 16:2646-55. https://doi.org/10.1158/1078-0432.ccr-10-0041.

24. Hoos A, Cordon-Cardo C. Tissue microarray profiling of cancer specimens and cell lines: opportunities and limitations. Lab Invest. 2001; 81:1331-8. 